\title{
Claudel et les sauterelles de l'Apocalypse de Jean : de l'image au symbole ou de la forme à la force
}

\author{
Claudia Jullien \\ Université de Besançon
}

L'imaginaire apocalyptique est sans doute l'un des plus anciens de l'humanité. Il est lié au mouvement du cosmos, à la dynamique de l'histoire des hommes et aux fantasmes du moi. Son imagerie symbolique articule dimension anthropologique et dimension sacrée. La Bible est parsemée d'apocalyptisme dont les grandes visions célestes offrent un sens premier qui s'ouvre sur un sens second, sens d'un sens qui prétend toucher aux événements terrestres et à leur devenir. Ces tableaux rendus impressionnants par leur avalanche de métaphores se présentent comme des révélations ou « apocalypses » et appartiennent à la littérature apocalyptique, connue des Hébreux sans doute dès le IVe siècle avant Jésus-Christ. On en trouve des exemples dans les livres d'Ézéchiel, d'Isaïe, de Jérémie. Le terme grec apokalypsis a donné son nom au dernier livre du second Testament. Au-delà de la signification générale du terme (dévoilement, révélation), la symbolique de la fin du monde, l'obsession des choses dernières, la question du mal et du jugement de Dieu ont fini par imposer leur dimension eschatologique et morale.

L'Apocalypse de Jean ( ${ }^{\text {er }}$ siècle de notre ère), texte éminemment symbolique selon la tradition religieuse à laquelle Claudel se veut fidèle, a comme originalité de se référer à la figure du Christ et d'entrer dans une apocalyptique chrétienne. La dimension littéraire du texte se double d'une dimension religieuse dont la richesse et le mystère sont entretenus par le déploiement de métaphores vertigineuses. C'est sur ce livre biblique que commencent, en 1928, les ouvres dites « exégétiques » de Claudel. Sa «Rêverie sur l'Apocalypse » débute en 1928 comme une promenade dialoguée et deviendra Au milieu des Vitraux de l'Apocalypse. Dans les années 1941-1942, les « Notes complémentaires sur l'Apocalypse » aboutiront à Paul Claudel interroge l'Apocalypse. Dans cet énorme ouvrage, les sauterelles apparaittront encore plus qu'ailleurs puisqu'elles surgissent trois fois dans ce que Claudel lui-même appelle le «Tableau des 


\section{Claudia Jullien}

Trompettes $\gg^{1}$ (p. 1004-1005, 1028-1030 et 1082-1083) et de manière pratiquement identique dans deux de ces textes. On retrouve aussi les sauterelles dans des textes moins connus comme celui de la Prophétie des oiseaux de 1939, ou dans les longs Excursus I et II ou Suppléments à l'Apocalypse de 1953-1954. Dans « Les Quatre animaux sages » (1933), texte rattaché à Figures et paraboles, elles apparaissent un instant à l'intérieur d'une vision tout autre et font l'effet d'un contrepoint.

\section{Dans le labyrinthe des métaphores}

On a déjà beaucoup écrit sur Claudel et l'Apocalypse, mais il m'a semblé, à relire certains textes, que l'image biblique des sauterelles du chapitre IX, apparemment absentes du Journal, ce qui est peut-être à remarquer, valait pourtant la peine d'un court détour. Claudel fait en effet de cette image le foyer d'une constellation dynamique de métaphores révélatrice d'une vision du monde à laquelle il est profondément lié. Essayons de suivre cette image ailée et bondissante à travers les textes en ne prétendant ni à une étude systématique et chronologique, ni à des découvertes inédites. Essayons simplement d'apprécier ce que cette image peut apporter à la connaissance de l'œuvre, de son écriture et de son auteur.

Selon sa méthode, Claudel explore l'image évocatrice d'abord à l'intérieur du champ interprétatif traditionnel que permet la lecture allégorique et " accommodatice " (terme officiel) appliquée depuis l'époque médiévale par les Pères de l'Église. Claudel en affectionne la liberté contrôlée. Par elle, il respecte la tradition chrétienne qui lit dans le premier Testament l'annonce et la présence du Christ tout en pouvant sortir, dans la mesure de sa fidélité générale à la doctrine, des sentiers battus. La thématique de la destruction qui précède et englobe le surgissement des sauterelles est, au premier abord, peu chargée de poésie. Elle fait partie d'un discours référentiel sans surprise si ce n'est l'élément familier et actualisant que Claudel y glisse :

Le chapitre IX commence par une étoile qui descend et à qui on donne la clef du Puits de l'Abîme, et alors il s'en échappe de la fumée comme d'une grande fournaise, comme qui dirait d'une usine, une fumée si noire qu'elle obscurcit le soleil et l'air de Dieu. Et ces nuages de fumée 
se changent en sauterelles qui ont le torturant pouvoir des scorpions. (Au milieu des vitraux, p. 128)

Affiliées au nom de leur roi Abaddon ou Apollyon, les sauterelles sécrètent la thématique traditionnelle de l'extermination ou de la destruction ( $A u$ milieu des vitraux, p. 105 et 129). Le jeu de mots que fait Claudel sur Apollon/Apollyon (destructeur) suscite un glissement qui donne au commentaire claudélien sa marque personnelle même s'il provient d'Eschyle (Agamemnon, vers 1081). Cela permet à Claudel d'étayer son texte en trois couches de métaphores selon une terminologie qu'il propose lui-même, entre sérieux et humour, dans $A u$ milieu des vitraux (ch. I, p. 108) : la strophe, l'antistrophe et la catastrophe. Ces termes qui rappellent le théâtre antique comportent des connotations esthétiques, morales et religieuses, la strophe correspondant au texte de la Bible, l'antistrophe, au monde en perdition, monde allant tout droit à la dernière étape, celle de la catastrophe. Cette ultime strate en particulier est la création personnelle de l'auteur qui greffe ses propres éléments sur le texte biblique. Ainsi, les « sauterelles dévoratrices de la substance auxquelles préside le roi Abaddon ou Apollyon [Ap $9,11]$ qui signifie Exterminateur » (ibid., p. 105), suggèrent au poète plusieurs couches de métaphores : «l'image des mauvaises pensées et la passion de l'analyse acharnée sur elle-même, l'âme retournée sur ellemême qui se regarde et qui se ronge » (ibid.). Claudel s'engage ainsi sur son grand cheval de bataille, à savoir la critique du rationalisme sur laquelle il va alors s'acharner d'un texte à l'autre par l'intermédiaire d'Apollon devenu Apollyon. Un peu plus loin, le couple cigales/ sauterelles fait son entrée dans la même constellation de métaphores. Les cigales (que l'on cherche en vain dans la Bible) sont les lecteurs stériles de livres eux aussi stériles, et les sauterelles évoquent livres et opinions dans le contexte dévalorisant d'une sorte de confusion mentale. On entre dans une interprétation étrangère au texte biblique et à dimension polémique :

Quand on a lu beaucoup de livres leur désaccord devient un accord, ou en tout cas une confusion, ils ne font plus tous ensemble qu'une rumeur indistincte et neutre, comme les cigales un jour d'été que l'on cesse au bout d'un peu de temps d'entendre. (ibid., p. 129) 


\section{Claudia Jullien}

Délivré des contraintes exégétiques, Claudel se déchaîne alors au cours d'un jeu de massacre dirigé contre le rationalisme desséchant dont il fait, à la moindre occasion, sa cible préférée. Il passe à l'attaque frontale :

Quelle meilleure comparaison que cet animal vide, cette bête à bruit, avec ses ailes en papier, et ces deux petits yeux cruels vissés de chaque côté d'un grand front idiot de penseur? Les couronnes d'or, pareilles à celles des distributions de prix, c'est la vanité ordinaire à tous les gratte-papiers. Les visages d'homme [Ap 9, 7], ça veut dire que ce sont des opinions humaines, chacune de ces sauterelles quand on la prend dans la main est comme une petite machine sèche dont toutes les pièces sont visibles, cela tient de l'automate, et tout à coup ça saute, ça vous fait au hasard dans la figure une espèce de pet-enl'air. (ibid.)2.

Comme on le constate, Claudel a entraîné son lecteur bien loin des rigueurs mallarméennes du « qu'est-ce que cela veut dire? " et il a beau faire le modeste ( Hélas, moi aussi, je ne suis qu'une pauvre sauterelle parmi les autres "), cela ne l'empêche pas de frapper fort contre le « poison» spirituel du rationalisme :

"La queue de scorpion il y a trois interprétations : ou c'est la seringue d'inoculation dont nous parlions tout à l'heure [p. 128 : allusion à « l'inoculation " des idées rationalistes et positivistes], ou c'est le tourment des questions insolubles, l'introspection, l'intelligence retournée sur elle-même et qui se dévore le ventre, ou c'est l'absurdité inhérente à toutes les théories humaines, la contradiction intérieure qui les tue au bout d'un certain temps suivant ce goût de suicide qu'on attribue au scorpion : ce blême intellectuel, pensionnaire de toutes les paillasses malpropres. » (p. 129)

Dans le champ de mire de Claudel on trouve ses ennemis ou frères ennemis, car Claudel peut très bien, religion mise à part, avoir des traits intellectuels appartenant à l'un ou à l'autre (plume critique ou sarcastique ou démesure des prises de position par exemple) : Voltaire, Anatole France, « les Belles-Lettres qui président aux ébats universitaires ", sans compter les « Neuf Muses pulvérulentes » qui, pour une fois, 
reçoivent un bon coup par derrière (p. 130) ! Claudel fulmine et décoche ses mots par la médiation des cigales qui n'ont rien à voir avec le texte biblique mais semblent évoquer de loin un frère en critique des princes de ce monde, ce La Fontaine qui savait lui aussi utiliser le pouvoir symbolique et provocateur des métaphores :

ces assis et ces imaginatifs sont des obsédés [...] Ils ont cette végétation derrière la tête de mauvaises imaginations dont ils ne peuvent se débarrasser.

- Voilà les cigales et cette assourdissante clameur qui s'élève des bibliothèques. Leur roi est Apollyon, qui veut dire destructeur [...] (ibid. p. 129).

C'est dans son grand commentaire de l'Apocalypse que Claudel atteint une sorte d'apogée péjorative des sauterelles. L'élaboration difficile du texte permet de suivre le fil embrouillé de sa composition grâce à un remaniement important relevé dans ses variantes et commenté par Michel Malicet dans son édition (voir Paul Claudel interroge l'Apocalypse, p. 1025-1033 et les notes). Il constate d'abord que Claudel a supprimé un long passage du texte initial (brouillon B) axé sur le problème du Mal, suppression qui deviendra ultérieurement le livre de Job. Il remarque aussi que le texte de remplacement, centré à nouveau sur les sauterelles, reprend un thème déjà développé précédemment (ibid., p. 1005 sq.) et qui reviendra encore plus loin, et en termes identiques, dans un passage postérieur existant déjà dans le brouillon et maintenu dans la copie. On peut en effet comparer les textes des pages 1026 sq. et 1081 sq. et constater l'importance des éléments communs. Dans une note de son édition (p. 1709), Michel Malicet constate que cet embarras des textes qui se font écho, parfois en se répétant, « illustre à la fois l'acharnement de Claudel à décrypter la vision, son admiration devant le spectacle offert et parfois son désespoir devant la tranquille opacité du texte ». Il semble aussi que ces difficultés traduisent, face à la résistance et à la dynamique du texte biblique, cet étonnant pouvoir poétique, ce génie de Claudel qui sait vivre, par la Bible, une expérience symbolique, et en extraire de nouvelles harmoniques dont il nourrit alors son propre chant pour le communiquer à son lecteur. 


\section{Vers la communication d'une force par l'émergence du symbole}

La première apparition des sauterelles dans le grand commentaire de Claudel s'inscrit dans le chapitre IV («Les sept trompettes ») et le paragraphe concernant la Cinquième trompette (p. 1003). On repère dans le passage une certaine démarche esthétique de Claudel qui fait traverser à la métaphore biblique ('image-plus' ou image par excès de la sauterelle réelle) un contexte où s'accumulent les images abstraites. Elles renforcent l'impression de mystère et, en même temps, elles participent d'un nouvel univers de mots et de sens. On passe du monde visible où les événements se déroulaient linéairement au monde invisible dégagé de la notion de temps :

$\mathrm{Du}$ fond de la matière, du fond de la conscience, il s'élève une fumée, c'est-à-dire une éruption volumineuse résultant d'une destruction et d'une libération de substance, qui obscurcit le soleil et l'air. Et cette vapeur de refus et de négation, perdition et déperdition, voici qu'elle devient vivante et active : plonge la main dedans, lecteur, plonge la main dans ton propre cœur! et tu en retireras une poignée de sauterelles.

On ne peut s'empêcher de penser à l'image du " nœud de vipères " (le roman de Mauriac qui porte ce titre est de 1932). Mais Claudel sait ménager les surprises en déroutant les mots (et le lecteur parfois) pour les conduire sur de nouveaux chemins où la métaphore, déjà porteuse d'un premier sens, devient alors le sens d'un sens autre sinon entièrement nouveau. Le monde visible disparaît et le monde invisible, monde intérieur de l'auteur, son état d'âme en quelque sorte, se substitue au monde visible et ouvre ainsi au sens premier un chemin vers un autre sens qui cependant a besoin du premier pour exister. Le chant claudélien se constitue à partir d'une communication souterraine dont l'opacité préserve le mystère et se mêle à l'état d'âme, ou, pour employer un terme un peu freudien, à la « sphère libidinale ${ }^{3}$. Dominant le champ syntagmatique de son propre texte, texte double mêlant dans sa linéarité images bibliques (couche externe) et métaphores personnelles (couche interne), Claudel peut évoluer librement de bas en haut de son champ paradigmatique, dans l'éclatement de l'enchaînement libre et substitutif des images. 


\section{The Claudel et les sauterelles de l'Apocalypse de Jean}

Il construit ainsi sa propre vision intérieure et révèle quelque chose de son moi profond : une véritable hantise de tout ce qui, en lui ou à l'extérieur de lui, s'oppose à Dieu :

Un visage d'homme, des cheveux de femme, une couronne d'or sur la tête, une queue de scorpion, quoi encore? il y intervient des éléments de lion et de cheval, et n'oublions pas pour enveloppe cette cuirasse dure et sèche. C'est que le péché en effet est une chose composite, et quand nous faisons le mal, c'est par tous les bouts, pour ainsi dire, de notre nature. La perversion de l'être que caractérise l'acte principal intéresse tout l'ensemble de notre machine. La couronne d'or sur la tête, c'est l'orgueil, mais cette détente, ce coup de reins qui nous projette tout à coup dans l'inconnu, c'est le désir aveugle, la folle imagination, l'impulsion des instincts de pardessous et de par-derrière. [...] Nous voici armés chevaliers! la voici équipée, cette sauterelle humaine qui résulte de l'hymen du diable avec le néant! (Paul Claudel interroge l'Apocalypse, p. 1004).

Quelques pages plus loin (ibid., p. 1083, texte déjà présent dans le brouillon et maintenu dans la copie comme nous l'avons mentionné plus haut), les sauterelles sont redevenues les instruments de la destruction, « une vermine autonome, une activité malfaisante et polymorphe », écrit Claudel. Mais, en établissant une comparaison entre les sauterelles destructrices et des « chevaux prêts pour la guerre » (Apocalypse IX, 7), le texte biblique permet le grossissement monstrueux des sauterelles que Claudel va encore renforcer à sa manière. Il concrétise cette monstruosité en évoquant le « portrait de la sauterelle géante de Transcaucasie » qu'il a pu voir dans l'Illustration (revue documentaire) et que l'on appelle en Martinique le «Cheval-Bon-Dieu " (Au milieu des vitraux, p. 128). Cette métaphore de substitution fait d'elle la création et l'instrument d'un Dieu terrifiant et justicier. Claudel, sous des dehors parfois anodins (ici le dialogue familier entre le Père et la Fille), fait œuvre de poète hanté par sa vision intérieure. À la faveur de ce malaxage des images, le poète (ou plutôt le " poëte ") entre et fait entrer le lecteur dans ce que P. Ricœur appelle "l'expérience symbolique ». Elle s'appuie sur le " travail de sens" produit ou sécrété par la métaphore et son organisation « en réseaux et 


\section{Claudia Jullien}

couches hiérarchiques »( $\mathrm{P}$. Ricœur, article cité, p. 158). La gigantomachie ouvre peut-être bien le texte sur un effroi qui se situe entre Éros et la mort (P. Ricœur, ibid.). On pourrait, semble-t-il, aller plus loin encore avec $\mathrm{P}$. Ricœur et envisager que Claudel se livre à un transfert de sens qui a pour conséquence de "révéler » de nouvelles significations. On retrouverait donc dans les commentaires bibliques, œuvres suscitant de nombreuses questions interdisciplinaires, un Claudel dont le symbolisme se situe au carrefour des sciences humaines :

Et, de fait, l'histoire des mots et celle de la culture semblent indiquer que si le langage ne constitue jamais que la couche superficielle de notre expérience symbolique, en retour, celle-ci ne nous devient accessible que dans la mesure où elle est formée, articulée, à un niveau linguistique et littéraire, les métaphores les plus insistantes se tenant en quelque sorte à la flexion de l'infra-structure métaphorique. (P. Ricœur, ibid.).

Dans « La prophétie des oiseaux » (1939), Claudel écarte un symbolisme trop concret ou trop simple (sauterelles = aéroplanes) et décoche encore ses flèches (à la manière aussi d'une autoflagellation ironique!) contre ce symbole de « la race exécrable des hommes de lettres " (p. 915). En fin de compte cependant, il faut remarquer que les sauterelles s'inscrivent à l'intérieur d'un mythe claudélien de la création qui emprunte des éléments théologiques pour les articuler selon une logique poétique :

Dans l'éblouissement d'un épouvantable coup de tonnerre nous recevons en plein visage une rafale de grêle et de feu! Quelle catastrophe! Le tiers de l'architecture angélique qui s'arrache et qui s'effondre, le tiers de la famille de Dieu qui abjure son Père et qui lui dit : Non! en plein visage! Une rupture, une hémorragie électrique propageant de tous côtés l'incendie et l'explosion, et dans le suspens de ce magnifique équilibre dont la clef est l'amour tout à coup l'intervention de la volonté particulière et du poids! ( $P$. Claudel interroge l'Apocalypse, p. 1027 et 1081). 
Mais l'on peut admettre, comme P. Ricœur, que la symbolique constitue « une réserve de sens dont le potentiel métaphorique reste toujours à dire " (article cité, p. 158), ceci étant valable également pour le texte biblique, une fois dépassée l'allégorie et l'interprétation traditionnelles. Les sauterelles des «Quatre animaux sages » (Figures et paraboles, édition critique d'Andrée Hirschi, Les Belles-Lettres, 1974, p. 191 sq.) témoignent de cette prolifération du symbole qui, dit P. Ricœur, « ne meurt jamais mais toujours se transforme » (article cité, p. 157). Dans Proverbes XXX, 27 qui sert de point de départ au texte claudélien de 1939 , on ne trouve nulle trace d'interprétation négative. Ici, la sauterelle est "sage ", même si le mot " dévastatrice " s'impose encore à la plume claudélienne par référence aux sauterelles destructrices réelles. Mais Claudel dépasse le texte biblique, dépasse le concret par l'abstrait, revient au concret et, dans ce tournoiement, entre dans l'expérience symbolique la plus intime : la sauterelle qui sait utiliser vent et instinct pour s'élever possède le nombre pour puissance... ( «l'instrument de sa puissance est le nombre ", écrit Claudel). D'abord orienté par le contexte biblique de la "sagesse » des sauterelles des Proverbes où elles ne jouent pas les trublions, le poète revient au réel : elles dévorent tout ce qui est vert, contrairement à Apocalypse IX, 4. Le symbole biblique dérouté par Claudel revient à Claudel et le lecteur peut apprécier alors l'invention herméneutique d'un " poëte "se laissant porter par une inspiration, un souffle dont les grandes ailes se déploient dans la démesure du cosmos, de son tempérament, de sa foi et de son art :

Je veux dire qu'elle [la sauterelle] doit s'arranger pour être, comme dit notre texte, universelle, c'est-à-dire catholique et pour couvrir à la fois sous le flot de son avancement irrésistible tout l'ensemble d'immenses territoires. Elle se divise comme d'elle-même en bataillons, sans autre méthode ou commandement que ce désir dévorateur. Tout ce qui est vert est sa proie : elle est prête à transformer chaque brin d'herbe en parole. (Figures et paraboles, « Les Quatre animaux sages », édition citée, p. 194)

Cette sauterelle qui transforme le monde en un livre où tout s'écrit en même temps, et qui ressemble parfois à un scorpion, n'est-elle pas finalement aussi un symbole de Claudel lui-même? 


\section{Claudia Jullien}

Le symbole est un fait de parole ou de discours doublé d'un élément qui puise sa force dans la vie du sujet. Il ne peut se transmettre que par les mots. On comprend alors la dimension vitale que pouvaient prendre pour Claudel ses commentaires exégétiques entremêlant les métaphores bibliques à la dimension symbolique la plus enracinée dans son tissu intime et son bouillonnement intérieur. L'expérience symbolique est peut-être tout aussi forte que l'expérience religieuse dans le sens où « le symbole [...] témoigne de l'enracinement premier du Discours dans la Vie. Il naît au point où Force et Forme coïncident. " (P. Ricœur, article cité, p. 153).

\section{Notes}

1 Pour les références et la pagination des œuvres et citations de Claudel, voir la récente édition critique dirigée par Michel Malicet, Paul Claudel, Le Poëte et la Bible, tome I, 1910-1946, Gallimard 1998.

2 L'allusion à la distribution des prix rend le texte transparent. C'est Renan, encore une fois, qui est visé. Voir le récit officiel de la conversion (CEuvres en prose, Gallimard, Bibliothèque de la Pléiade, 1965, p. 1009).

3 Paul Ricœur, "Parole et symbole ", in Le Symbole, Colloque international, 4-8 février 1974, Revue des Sciences Religieuses, no 1-2 de Janvier-Avril 1975, p. 142-161 : «Le fait opaque c'est que les symboles sont enracinés dans des champs offerts eux-mêmes à une investigation dispersée. Que la psychologie prenne le rêve pour paradigme des représentations substituées et déguisées, présuppose que l'on a d'abord pris en considération le sommeil comme contexte de l'activité onirique. L'image poétique n'est pas moins liée à une manière globale de se comporter que l'allemand appelle dichten, 'poétiser'. Et aurions-nous des symboles religieux si l'homme ne se livrait à des conduites très complexes et très spécifiques visant à évoquer, à implorer, éviter des forces surnaturelles qui habitent au plus profond de l'existence humaine, la surpassent et la dominent? De multiples manières, l'activité symbolique manque d'autonomie; c'est une activité liée. Et c'est la tâche de plusieurs sciences indépendantes de mettre à nu les liens par lesquels la fonction symbolique se rattache à telle ou telle activité non symbolique ou pré-linguistique ». (p. 151-152) 
The Claudel et les sauterelles de l'Apocalypse de Jean

4 Le Dictionnaire de la Bible dans la Littérature française - figures, thèmes, symboles, auteurs (à paraître aux éditions Vuibert et signé du même auteur) offre de nombreux exemples montrant la dynamique de l'expérience symbolique aux prises avec la culture biblique. 